In der Rubrik „Literatur kompakt" werden die wichtigsten Originalarbeiten aus der internationalen Fachliteratur referiert.

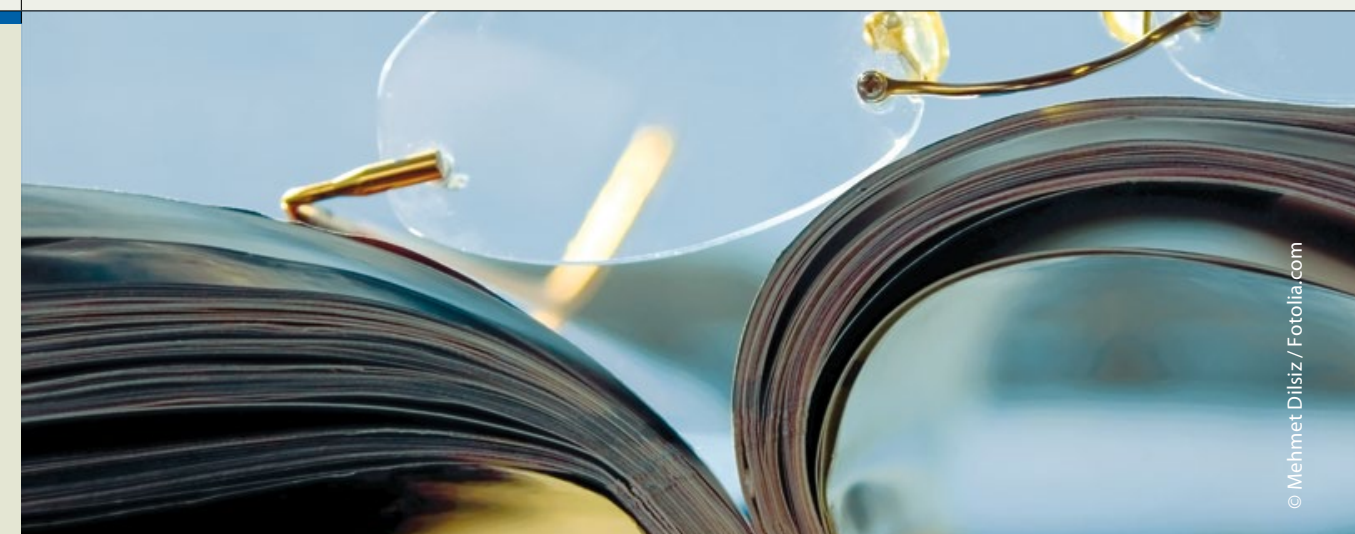

\section{TRPM8-Agonisten lindern Pruritus bei trockener Haut}

\section{Gegen chronischen Juckreiz infolge von trockener Haut könnte eine Kombination aus zwei TRPM8-Agonisten helfen. Eine Pilotstudie.}

$P_{d}^{a}$ atienten mit chronischem Pruritus, der durch trockene Haut bedingt ist, verwenden häufig Menthol-haltige Emollenzien. Durch Menthol wird der Kälterezeptor TRPM8 aktiviert und der Juckreiz unterdrückt. Da Menthol Hautirritationen auslösen kann und seine Wirkung vor allem nur wenige Minuten anhält, sind alternative TRPM8-Agonisten mit längerer Wirkdauer wünschenswert. Eine Kombination aus zwei solcher Agonisten - (1R,2S,5R)-N-(2-(2-Pyridinyl)ethyl)-2-ispropyl-5-methylcyclohexancarboxamid (CHC) und Menthoxypropandiol (MPD) - wurde jetzt in einer von Beiersdorf gesponsorten, klinischen Studie geprüft.

An der randomisierten Doppelblindstudie beteiligten sich 70 Patienten mit chronischem Pruritus bei trockener Haut. Sie sollten sich zweimal täglich eincremen, entweder mit der Kombination aus $\mathrm{CHC}$ und MPD in einer $\mathrm{O} / \mathrm{W}$ Lotion $(\mathrm{n}=36)$ oder mit dem Träger allein $(n=34) .59$ Patienten blieben bis zum Studienende nach vier Wochen dabei.

$\mathrm{Zu}$ diesem Zeitpunkt gaben signifikant mehr Patienten in der CHC/MPDals in der Kontrollgruppe eine Besserung des Pruritus an (79\% vs. $47 \%)$, damit war das primäre Studienziel erreicht. Auch gemessen an einer verbalen $\mathrm{Ra}$ ting-Skala und der prozentualen Verbesserung des Juckreizes waren die Patienten mit der wirkstoffhaltigen Lotion im Vorteil. Die Intensität des Pruritus ging allerdings mit beiden Behandlungen

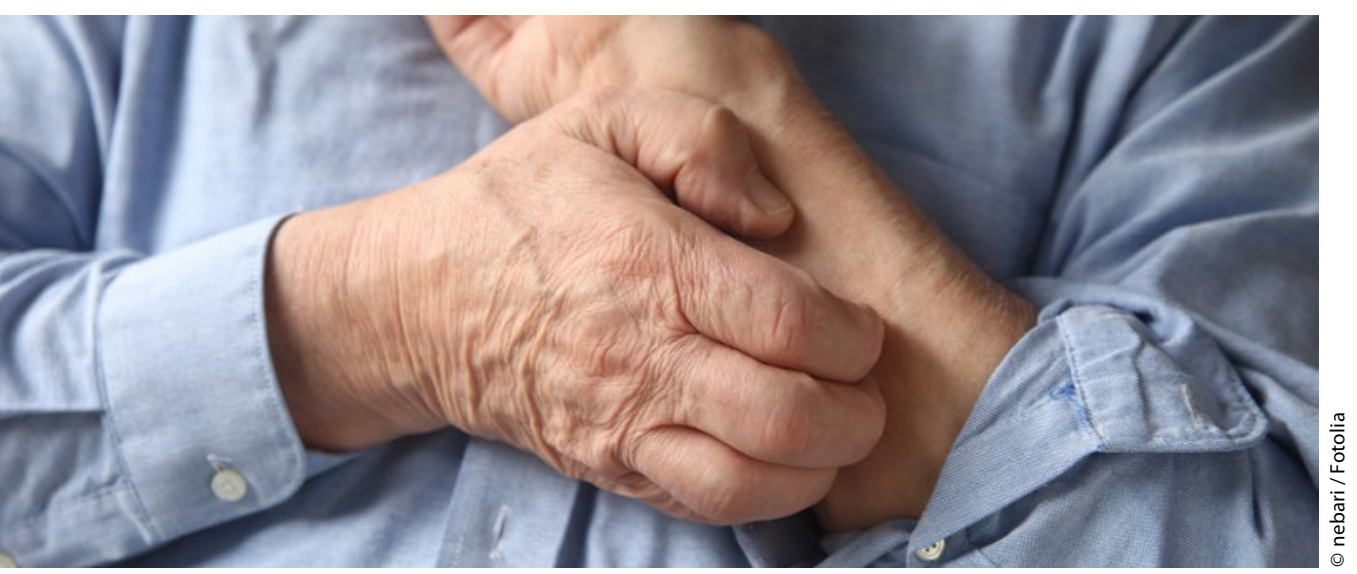

deutlich zurück (CHC/MPD: NRS von 6,7 auf 4,3; Träger: NRS von 5,9 auf 4,5), der Unterschied zwischen den Gruppen war nicht signifikant. Die gesundheitsbezogene Lebensqualität verbesserte sich ebenfalls in beiden Gruppen, hier lag die $\mathrm{CHC} / \mathrm{MPD}$-Gruppe wieder vorn.

Mit der CHC/MPD-Lotion berichteten bis zu $84 \%$ der Patienten über ein lang anhaltendes und starkes Kühlungsgefühl, das waren signifikant mehr als mit der Lotion ohne Wirkstoff, wo maximal $57 \%$ eine Kühlung wahrnahmen.

Nebenwirkungen traten in der $\mathrm{CHC} /$ MPD-Gruppe häufiger auf $(67 \%$ vs. $15 \%)$. Diese bestanden in einem zu starken Kältegefühl oder Brennen. Kontaktallergien gab es nicht, es wurde auch keine Tachyphylaxie beschrieben. Rauigkeit und Hydratation der Haut verbesserten sich ohne Unterschied in beiden Gruppen.

Fazit: Die Kombination der beiden TRPM8-Agonisten habe langanhaltende, klinisch relevante und im Vergleich zum reinen Träger signifikant bessere antipruriginöse und kühlende Effekte, so die Studienautoren. Sie sei damit ein möglicher Behandlungsansatz für Patienten mit chronischem Pruritus bei trockener Haut, die auf reine Emollenzien nicht ansprechen. Die Ergebnisse der Studie müssten aber als vorläufig betrachtet werden, außerdem müsse nach einer wirksamen CHC/MPD-Konzentration mit minimaler Nebenwirkungsrate gesucht werden.

Dr. Beate Schumacher

Ständer S et al. Novel TRPM8 agonist cooling compound against chronic itch: results from a randomized, double-blind, controlled, pilot study in dry skin. J Eur Acad Dermatol Venerol 2017; 31: 1064-8 